\title{
NEAR-INFRARED LINE IMAGING OF THE CIRCUMNUCLEAR STARBURST RING IN NGC 7771
}

\author{
J. Reunanen and J. K. Kotilainen \\ Tuorla Observatory, University of Turku, Väisäläntie 20, FIN-21500 Piikkiö; reunanen@astro.utu.fi, \\ jkotilai@stardust.astro.utu.fi \\ S. LAINE \\ Department of Physical Sciences, University of Hertfordshire, College Lane, Hatfield, Herts. AL10 9AB, \\ U.K.; seppo@star.herts.ac.uk \\ AND \\ S. D. RYDER \\ Joint Astronomy Centre, 660 N. A'Ohoku Place, Hilo, HI 96720; sryder@jach.hawaii.edu \\ Accepted for publication in ApJ
}

\begin{abstract}
We present high spatial resolution near-infrared broad-band $J H K$ images and, for the first time, $\operatorname{Br} \gamma$ $2.1661 \mu \mathrm{m}$ and $\mathrm{H}_{2}$ 1-0 S(1) $2.122 \mu \mathrm{m}$ emission line images of the circumnuclear star forming ring (major axis diameter $7^{\prime \prime}=2 \mathrm{kpc}$ ) in the starburst galaxy NGC 7771. These data are used to investigate the morphology and extinction of the starburst ring and to study its star forming properties and history by comparing the observed quantities with an evolutionary population synthesis model.

The clumpy morphology of NGC 7771 varies strongly with wavelength, due to the combination of extinction (for which we derive an average value of $\mathrm{A}_{V}=2.8$ ), emission from hot dust and red supergiants, and several stellar generations in the ring. Also, the ellipticity and the position angle of the ring depend on the wavelength.

The starburst ring in NGC 7771 exhibits small $\operatorname{Br} \gamma$ equivalent widths. Assuming a constant star formation model with $\mathrm{M}_{u}=100 \mathrm{M}_{\odot}$ results in very long lifetimes of the star forming regions (up to 1 Gyr), in disagreement with the clumpy near-infrared morphology and the observed radio spectral index of NGC 7771. This situation is only slightly remedied by assuming a reduced upper mass cutoff $\left(\mathrm{M}_{u}\right.$ $\left.=30 \mathrm{M}_{\odot}\right)$, resulting in ages between 8 and $180 \mathrm{Myr}$. We prefer an instantaneous star formation model with $\mathrm{M}_{u}=100 \mathrm{M}_{\odot}$ which can explain the derived $\operatorname{Br} \gamma$ equivalent widths if a single starburst occurred 6-7 Myr ago. The main excitation mechanism of the molecular gas, based on the observed $\mathrm{S}(1) / \mathrm{Br} \gamma$ ratio, appears to be excitation by UV radiation from hot young stars. We derive $\mathrm{M} \simeq 1900 \mathrm{M}_{\odot}$ for the mass of the excited $\mathrm{H}_{2}$.
\end{abstract}

Subject headings: galaxies: individual (NGC 7771) - galaxies: starburst - infrared: galaxies - stars: formation

\section{INTRODUCTION}

NGC 7771 (UGC 12815) is a very luminous nearby ( $\mathrm{v}_{\text {sys }}$ $\left.=4256 \mathrm{~km} \mathrm{~s}^{-1}\right)$ highly inclined $\left(\sim 69^{\circ}\right.$, Smith et al. 1999; hereafter S99) SBa galaxy, which forms a well-known pair with NGC 7770. At the distance of $56.7 \mathrm{Mpc}\left(\mathrm{H}_{0}=75\right.$ $\mathrm{Mpc}^{-1} \mathrm{~km} \mathrm{~s}^{-1}$ ) $1^{\prime \prime}$ corresponds to $280 \mathrm{pc}$. For a large scale view of the pair, see e.g. S99 (their Fig. 1). The optical morphology of NGC 7771 is strongly affected by heavy extinction and prominent dust lanes in the bar. NGC 7771 is interacting with NGC 7770 and NGC 7771A, as indicated by the $\mathrm{H} \alpha$ rotation curve (Keel 1993) and the HI velocity field (Nordgren et al. 1997).

There is no sign of a Seyfert nucleus in NGC 7771. Most of the luminosity of NGC 7771 arises from a massive circumnuclear starburst, which was probably triggered by the interaction with NGC 7770. Nuclear star formation (SF) in NGC 7771 is concentrated in an elliptical ring with a major axis of $\sim 7^{\prime \prime}$ (2 kpc), surrounding the starburst nucleus. This ring is clumpy and contains several emission regions both in radio (Neff \& Hutchings 1992) and nearinfrared (NIR) continuum (S99).

S99 noted an anticorrelation between the radio and NIR peaks, and explained this by two starburst episodes $\sim 10$
Myr (radio regions) and $\sim 4-8 \mathrm{Myr}$ ago (NIR regions). A lower limit (4 Myr) for the age of the starburst has also been estimated from the dominant presence of red supergiants (RSG), based on NIR CO spectroscopy (Smith et al. 1996). The inner regions of NGC 7771 are also much more luminous in the $K$-band than those in normal galaxies (S99), which can be explained easily if there is a substantial population of RSGs in NGC 7771.

We present high resolution NIR $J H K$, and for the first time, $\mathrm{Br} \gamma$ and $\mathrm{H}_{2}$ emission line images of the circumnuclear ring in NGC 7771. Br $\gamma$ emission arises from HII regions surrounding young, hot $\mathrm{OB}$ stars, while $\mathrm{H}_{2}$ 1-0 $\mathrm{S}(1)$ emission (hereafter $\mathrm{S}(1)$ ) arises from hot molecular gas and traces the material available for SF. The ratio between these two lines can give clues about the excitation mechanism(s) of the hot molecular gas (Puxley, Hawarden \& Mountain 1990).

Our paper is organized in the following way. In Section 2 we discuss the observations and data reduction. In Sections 3 and 4 we discuss the evolutionary model used and morphology of the circumnuclear ring and constrain its star forming properties, stellar populations and star forming history by a detailed comparison among the NIR tracers and also compare them with the radio emission. 
Conclusions are presented in Section 5 .

\section{OBSERVATIONS AND DATA REDUCTION}

Observations were made in September 1998 with the $3.8 \mathrm{~m}$ United Kingdom Infrared Telescope (UKIRT) on Mauna Kea, Hawaii. Seeing during the nights was FWHM $0{ }^{\prime \prime} 6-0.7$. We used the $256 \times 256$ pixel IRCAM3 camera, with pixel size $0^{\prime \prime} 281 \mathrm{px}^{-1}$ and field of view $\sim 70^{\prime \prime} \times 70^{\prime \prime}$. NGC 7771 was imaged in the $\operatorname{Br} \gamma 2.1661 \mu \mathrm{m}$ and $\mathrm{H}_{2} 1-0$ $\mathrm{S}(1) 2.122 \mu \mathrm{m}$ spectral lines and in the $J H K$ bands. For the emission line observations, we used cooled $(T=77 \mathrm{~K})$ narrow-band filters and a Fabry-Perot etalon with spectral resolution $\sim 400 \mathrm{~km} \mathrm{~s}^{-1}$ and equivalent width $0.0038 \mu \mathrm{m}$.

For both lines, 5 one-minute observations were made at each on-line wavelength (OL) and at the nearby blue (BC) and red (RC) continuum, following the sequence OL$\mathrm{BC}-\mathrm{OL}-\mathrm{RC}$ and moving the telescope by $8^{\prime \prime}$ between the individual integrations. A dark frame was obtained for each sequence. For the $J H K$ bands, 5 one-minute integrations were taken both centered on NGC 7771 and on sky $5^{\prime}$ east of NGC 7771, moving the telescope as in the line observations.

$I R A F^{1}$ was used to reduce and analyze the observations. For the line images, a normalized flatfield was made from linearized and dark-subtracted galaxy images. Galaxy frames were then divided by the flatfield and skysubtracted using the median of the 5 images obtained at the same wavelength. Frames were aligned and merged into one on-line image and two continuum images. Since the shape of the nucleus varies with wavelength, $J H K$ band images were first registered using a foreground field star. The emission line and $K$ band images were then registered with respect to each other by using the nucleus prior to subtracting the continuum in order to avoid possible rotation and alignment errors. The final registration of the images is accurate to within a fraction of a pixel. Finally, the continuum images were scaled with the continuum/line ratio measured from observations of a standard star (HD 225023), combined and subtracted from on-line images, and the final line images were then flux calibrated. The total on-line integration time was 40 minutes for $\mathrm{Br} \gamma$ and 20 minutes for $\mathrm{S}(1)$. Broad-band images were linearized, dark-subtracted, flatfielded using a flatfield made from sky images and sky-subtracted using the median of 5 sky images. FS $29(K=13.346)$ from the Faint Star Catalogue of UKIRT, was used for flux calibration and the observed fluxes were corrected for Galactic extinction and redshift of NGC 7771 (K-correction).

The wavelength dependence of the Fabry-Perot was corrected for by dividing the measured fluxes by an Airy function (see e.g. Bland-Hawthorn 1995). Both the velocity field of NGC 7771 and the shift in the transmitted wavelength across the array need to be considered. A high resolution velocity field for NGC 7771 has not been published, but in a lower resolution data (Nordgren et al. 1997) the velocity contours are nearly perpendicular to the major axis of the elliptical ring, so the velocity field in the ring was approximated by the 1D H $\alpha$ longslit-data (Keel 1996). The effect of linewidth on the emission line fluxes has not been corrected for because the shape of the lines in the ring is not known. The observed line emission is weak, so in order to enhance the $\mathrm{S} / \mathrm{N}$, all images were smoothed to $1^{\prime \prime}$ resolution.

\section{MORPHOLOGY}

The inclined star forming ring with a major axis of $\sim$ $7^{\prime \prime}\left(2 \mathrm{kpc}\right.$ at $\left.\mathrm{PA} \simeq 90^{\circ}\right)$ is clearly visible in the $K$-band image (Figure 11). The nucleus in the middle of the ring is brighter than the circumnuclear regions. In the $H$-band and especially in the $J$-band, the morphology is different. The southeastern part of the ring is significantly more conspicuous in the $J$-band than in the $K$-band and the nucleus appears to merge with the northern part of the ring. In the east the ring is weaker than in the west in all the broad-band images. These differences in morphology are also clearly visible in the $J-H$ color map (Figure 2), where the southern and southwestern parts of the ring are redder than other parts, probably due to extinction, RSGs and hot dust.

Although the western part of the ring is weaker in the $K$-band, almost all $\mathrm{Br} \gamma$ emission arises from there (Figure 11. The eastern part of the ring may be older than the western part and supernova (SN) remnants may already have dispersed the gas clouds. Because RSGs appear at a later stage in the starburst evolution (Leitherer \& Heckman 1995) and then dominate the $K$-band emission, the eastern part may indeed be older. Even in the west, the $K$-band peaks do not correlate with the $\operatorname{Br} \gamma$ peaks, but seem to indicate older starburst regions. There is no $\mathrm{Br} \gamma$ emission source exactly at the nucleus, but $\sim 0^{\prime \prime} 4$ to the north of the nucleus there is a strong $\mathrm{S}(1)$ source. The position of this strong $\mathrm{S}(1)$ peak is affected by smoothing. In the unsmoothed image the peak pixel is coincident with the nucleus, but emission becomes fainter much faster to the south than to the north. This may be due to extended emission or an additional emission region north of the nucleus. We believe that the $\mathrm{S}(1)$ region marked $\mathrm{N}$ is really the nuclear emission which has shifted north during the smoothing. The position of other emission regions is unaffected by smoothing. The $\mathrm{S}(1)$ emission in the ring is distributed more broadly than the $\mathrm{Br} \gamma$ (Figure 1), especially in the east, delineating a more complete ring than what is seen in $\operatorname{Br} \gamma$. Neither the correlation between the $\mathrm{S}(1)$ and $K$-band peaks, nor between the $\mathrm{S}(1)$ and $\mathrm{Br} \gamma$ peaks is perfect, but there is a fair correlation between $\mathrm{S}(1)$ and $J-H$.

Although the ring is incomplete in $\operatorname{Br} \gamma$, it appears to follow an ellipse with semi-major and semi-minor axes of $a \simeq 3^{\prime \prime} \cdot 2$ and $b \simeq 1^{\prime \prime} \cdot 2$ at $\mathrm{PA} \simeq 69^{\circ}$. This ellipse is different from that in the $K$-band $\left(a \simeq 2^{\prime \prime} .8\right.$ and $b \simeq 1$.'2 at $\left.\mathrm{PA} \simeq 90^{\circ}\right)$, but it is consistent with the ellipse in radio emission $\left(a \simeq 2^{\prime \prime} .75\right.$ and $b \simeq 1^{\prime \prime} .25$ at $\mathrm{PA} \simeq 73^{\circ}$; S99). The $\mathrm{S}(1)$ emission appears to arise from a ring similar to the $\operatorname{Br} \gamma$ emission. The $K$-band ellipticity may be different since emission in the $K$-band is dominated by RSGs, whereas $\operatorname{Br} \gamma$ and radio continuum give the orientation of the younger starburst ring which can be affected by gas dynamics e.g. in a barred potential.

We compared our $\mathrm{Br} \gamma$ images and the radio images of S99 with after registering the $\operatorname{Br} \gamma$, radio and $\mathrm{K} K$-band

\footnotetext{
${ }^{1}$ IRAF is distributed by the National Optical Astronomy Observatories, which are operated by the Association of Universities for Research in Astronomy, Inc., under cooperative agreement with the National Science Foundation.
} 
images using Fig. 4 of S99. There is a reasonable correlation between the $\operatorname{Br} \gamma$ and the radio emission to the south and southwest of the nucleus (to within 0.'2). Elsewhere this correlation is worse, as the strongest $\operatorname{Br} \gamma$ peaks do not have radio counterparts to within a distance of $1^{\prime \prime}$. In general, $\mathrm{Br} \gamma$ correlates better with radio than with the NIR continuum. Morphological differences between the $\mathrm{Br} \gamma$, radio and NIR continuum images are probably due to differences in the ages of the emitting regions, possibly indicating several star forming epochs. A similar anticorrelation between the radio and NIR continua has also been detected in several other starburst systems, e.g. M82 (Golla, Allen \& Kronberg 1996) and NGC 253 (Sams et al. 1994; Ulvestad \& Antonucci 1997).

\section{STAR FORMING PROPERTIES}

We detected 9 emission regions defined by the $\operatorname{Br} \gamma$ above $3 \sigma$ level. Furthermore, the region $\mathrm{N}$, located at the nucleus, has no $\mathrm{Br} \gamma$ but strong $\mathrm{S}(1)$ emission. The photometric apertures used were selected to avoid overlap between neighboring regions, but to include as much of the $\operatorname{Br} \gamma$ emission as possible (regions 1-9). The smallest distance between the nearest emission regions is 1 .'2, which is larger than the resolution of the smoothed images $\left(1{ }^{\prime \prime} 0\right)$, and much larger than the seeing during the observations $\left(0^{\prime \prime} 6\right)$. Note that there is some overlap between region $\mathrm{N}$ and region 3. The smallest regions consist of only a few pixels, but these regions have radio counterparts (S99) and are therefore probably real. Furthermore, as all the regions consist of more than one pixel in unsmoothed image, they are unlikely be affected by cosmic rays or other defects. Most of the regions are also identifiable when $\operatorname{Br} \gamma$ frames were reduced separately in two halves. The aperture diameters used, the values of the Airy function, the observed and dereddened $\operatorname{Br} \gamma$ and $\mathrm{S}(1)$ fluxes and the $J H K$ colors for the emission regions are given in Table 1 .

To interpret the star forming properties of NGC 7771, we have used the evolutionary model of Leitherer et al. (1999; hereafter L99), which predicts various NIR, optical and UV spectral features as a function of metallicity, initial mass function (IMF), lower and upper mass cutoff and age, for the limiting cases of instantaneous star formation (ISF) and a constant star forming rate (CSFR).

The equivalent width (EW) of hydrogen recombination lines is sensitive to the age of the starburst. The $\mathrm{Br} \gamma$ EW was estimated by subtracting a smooth galaxy model from the $K$-band image and dividing the $\operatorname{Br} \gamma$ fluxes with the fluxes in the subtracted $K$-band image. The L99 models predict the number of ionizing photons, $\mathrm{N}\left(\mathrm{H}^{0}\right)$, which can also be estimated from the $\mathrm{Br} \gamma$ flux as $\mathrm{N}\left(\mathrm{H}^{0}\right)\left[s^{-1}\right]$ $=7.63 \times 10^{13} \mathrm{~L}_{B r \gamma}\left[\mathrm{erg} \mathrm{s}^{-1}\right]$ (Leitherer \& Heckman 1995). The number of ionizing photons allows us to evaluate the mass of recently formed stars (in ISF) or the star forming rate (in CSFR) via an assumed IMF. $\mathrm{N}\left(\mathrm{H}^{0}\right)$ can also be estimated from the thermal radio emission of HII regions (S99): $\mathrm{N}\left(\mathrm{H}^{0}\right)=7.1 \times 10^{49} \mathrm{D}^{2} \nu^{0.1} \mathrm{~T}_{e}^{-0.76} \mathrm{~S}_{t h}$, where $\mathrm{D}$ is the distance in Mpc, $\nu$ is the frequency in $\mathrm{GHz}, T_{e}$ is the electron temperature in $10^{4} \mathrm{~K}$ and $S_{t h}$ is the thermal radio flux density in mJy. Finally, the SN rate predicted by the L99 models can be compared with that derived from the nonthermal radio emission (Condon \& Yin 1990): $\mathrm{L}_{N T} \sim$ $1.3 \times 10^{23} \nu^{-\alpha} \mathrm{v}_{S N}$, where $L_{N T}$ is the nonthermal radio luminosity in $\mathrm{W} \mathrm{Hz}^{-1}, \nu$ is the frequency in $\mathrm{GHz}, \alpha$ is the spectral index of nonthermal radiation and $\mathrm{v}_{S N}$ is the $\mathrm{SN}$ rate in $\mathrm{yr}^{-1}$.

Since we do not have enough data for more detailed modeling, we have selected two models in what follows: (1) ISF with $M_{u}=100 \mathrm{M}_{\odot}$ and $\alpha=2.35$ and (2) CSFR with $M_{u}=30 \mathrm{M}_{\odot}$ and $\alpha=2.35$. We have assumed solar metallicity.

The extinction towards the ionized sources was estimated from the $\mathrm{Br} \gamma / \mathrm{H} \alpha$ ratio. $\mathrm{H} \alpha$ imaging has not been published for NGC 7771. Veilleux et al. (1995) give an $\mathrm{H} \alpha$ flux of $5.9 \times 10^{-14} \mathrm{ergs} \mathrm{s}^{-1} \mathrm{~cm}^{-2}$ in an aperture of $\sim 7^{\prime \prime} \times 2^{\prime \prime}$ $(2 \mathrm{kpc} \times 0.55 \mathrm{kpc})$ that includes most of the ring. We derive $A_{V}=2.8$ for the ring using the $\mathrm{Br} \gamma / \mathrm{H} \alpha$ ratio, Landini et al. (1984) extinction law $\left(A \propto \lambda^{-1.85}\right)$ and a foreground dust screen. Similar extinction values were also derived by Davies, Alonso-Herrero \& Ward (1997), who suggested an average $A_{V}=2.3$ for the central starbursting regions based on the Balmer decrement. If the dust and the emitting gas are mixed we derive $\mathrm{A}_{V}=4.0$. S99 derived much greater $A_{V}=5.2$ from the $\mathrm{H} \alpha / \mathrm{H} \beta$ ratio, and they suggested an even greater extinction $\left(A_{V}=6.5\right)$ towards the ionized gas from the radio/ $\mathrm{H} \alpha$ ratio. Higher extinction $\left(\mathrm{A}_{V}>5 \mathrm{mag}\right)$ is also suggested by the IR/blue luminosity ratio. Extinction probably varies significantly in the ring (see Figure 2 for variations in extinction towards the continuum sources), but due to the lack of a high resolution $\mathrm{H} \alpha$ image, we use the average extinction value $\mathrm{A}_{V}=2.8$ mag in what follows.

To provide a check for the extinction, we estimated the extinction towards the continuum sources in the ring from the observed $J H K$ colors compared to the average $J-H$ color of unobscured spiral galaxies $(<J-H\rangle=0.75,<H-$ $K>=0.22$; Glass \& Moorwood 1985) assuming that only extinction affects the colors. The observed $J-H$ colors (Figure 3) of the ring are redder than the areas inside or outside the ring. From the NIR colors we derive a slightly smaller extinction $\left(\mathrm{A}_{V}=0.5-3.9\right.$, average $\left.\mathrm{A}_{V}=2.2\right)$ than that measured from the recombination line ratios, indicating that RSGs responsible for the NIR continuum emission are located outside the HII regions surrounding the OB stars. Alternatively, this result may also be a selection effect: the most heavily reddened regions may not be detectable in our images, and thus we are biased towards the low-extinction regions. The southern half of the ring is redder than the northern half (Figure 2), and therefore extinction is probably greater in the south, as was noted by Davies et al. (1997).

The exact value of the extinction does not affect the $\mathrm{EW}$ of $\mathrm{Br} \gamma$ (ie. age) if there is no differential extinction between $\operatorname{Br} \gamma$ and continuum sources, but it does scale up or down the properties derived from the luminosity of $\operatorname{Br} \gamma$, including mass, $\mathrm{SFR}, \mathrm{N}\left(\mathrm{H}^{0}\right)$ and $\mathrm{SN}$ rate. Our method of analysis, however, depends on the details of the modeling. Real physical starbursts have a finite duration, so the ages derived for ISF are really lower limits, as the EW of $\mathrm{Br} \gamma$ grows as a function of the burst duration for a given age. The observed CO indices (Smith et al. 1996; Ridgway, Wynn-Williams \& Becklin 1994) together with the EW of $\mathrm{Br} \gamma$ can constrain the duration of the burst (Puxley, Doyon \& Ward 1997). The exponential decaying time of the burst may have been 1-5 Myr (Puxley et al. 1997, their Fig. 3), or alternatively $\sim 20 \mathrm{Myr}$, in which case the burst started $\sim 50 \mathrm{Myr}$ ago. 
The mass of the excited $\mathrm{H}_{2}$ can be estimated by following the procedure of Meaburn et al. (1998). The average surface brightness of $\mathrm{S}(1)$ in an 8 . $4(2.4 \mathrm{kpc})$ aperture is $6.4 \times 10^{-13} \mathrm{~W} \mathrm{~cm}^{-2} \mathrm{sr}^{-1}$ and assuming $\mathrm{T}_{v i b}=2000 \mathrm{~K}$ we derive $1740 \mathrm{M}_{\odot}$ for the mass of the excited $\mathrm{H}_{2}$. This value must be multiplied by $\sim 1.1$ for the Fabry-Perot calibration and by a small, unknown factor for the linewidth, which may be broader than the passband width of the FabryPerot $\left(\sim 400 \mathrm{~km} \mathrm{~s}^{-1}\right)$. The resulting value $\sim 1900 \mathrm{M}_{\odot}$ may be compared with the Seyfert galaxies NGC $3079\left(800 \mathrm{M}_{\odot}\right.$, Meaburn et al. 1998) and NGC $3227\left(250 \mathrm{M}_{\odot}\right.$, Fernandez et al. 1999). In all the cases the available mass of HI, as derived from the CO emission, is several magnitudes higher. The peak surface density of excited hydrogen is $1.9 \times 10^{21} \mathrm{~m}^{-2}$.

The $\mathrm{S}(1) / \mathrm{Br} \gamma$ ratio can give clues about the excitation mechanism (Puxley et al. 1990). The observed ratio of the emission regions in the south and southeast (0.71-0.95) are consistent with UV excitation by young stars. Elsewhere in the ring the ratio is slightly larger (1.30-2.10), indicating perhaps a relatively larger contribution from shock excitation, possibly due to supernovae or stellar winds. Alternatively, gas clouds may be denser, or the upper mass cut-off higher in these regions. This seems more plausible, because there is no evidence of lower SN rate in the south (Table 2; S99). The only emission region outside the ring (4) has the lowest value of $\mathrm{S}(1) / \operatorname{Br} \gamma(0.61)$.

We have detected the nucleus in $\mathrm{S}(1)$ but not in $\mathrm{Br} \gamma$, which is also the case in Seyfert galaxies NGC 1097 and NGC 6574 (Kotilainen et al. 1999). But unlike those galaxies, the nucleus is not redder than its surroundings. Thus, the extinction in the nucleus may be relatively low. The absence of $\operatorname{Br} \gamma$ (massive young stars) in the nucleus can be explained if the nucleus is either too old to harbor OB stars, or the star formation has not yet begun there.

The EW of $\mathrm{H} \alpha$ in the ring is $\sim 10-80 \AA$ (Davies et al. 1997), which according to the L99 standard model $\left(\alpha=2.35, \mathrm{M}_{u}=100 \mathrm{M}_{\odot}\right)$ corresponds to an age range $6.2-$ 11.6 Myr (ISF) or ages $>1$ Gyr (CSFR). In the ISF model, the age derived from the $\mathrm{EW}$ of $\operatorname{Br} \gamma(5.5-54 \AA)$ is 5.86.8 Myr, which would indicate copious numbers of RSGs. Their presence within the inner regions of NGC 7771 seems indeed probable because of the observed deep NIR CO absorption and the highly enhanced $K$-band luminosity (Smith et al. 1996; S99). With the ISF model and using the $\mathrm{Br} \gamma$ emission, the masses of the star forming regions are $0.3-3.2 \times 10^{7} \mathrm{M}_{\odot}\left(\right.$ average $\left.1.5 \times 10^{7} \mathrm{M}_{\odot}\right)$ and SN rates are $0.3-3.3 \times 10^{-2} \mathrm{yr}^{-1}$ (average $0.015 \mathrm{yr}^{-1}$ ). With the CSFR model and a reduced upper mass cutoff $\left(\mathrm{M}_{u}=30\right.$ $\mathrm{M}_{\odot}$ ), as suggested by Davies et al. (1997), the ages of several regions remain large, $>100 \mathrm{Myr}$. Such old ages are improbable, as single molecular clouds are unlikely to survive more than $\sim 40$ Myr (Blitz 1991). The total mass of the star forming regions is large, $\mathrm{M} \simeq 3 \times 10^{8} \mathrm{M}_{\odot}$, which is, however, much less than the total available molecular gas mass derived from the CO luminosity, $\mathrm{M}_{H_{2}} \simeq 9 \times 10^{9} \mathrm{M}_{\odot}$ (Sanders, Scoville \& Soifer 1991). With the CSFR model, the star forming rate of individual regions is $0.2-0.7 \mathrm{M}_{\odot}$ $\mathrm{yr}^{-1}$ (average $0.4 \mathrm{M}_{\odot} \mathrm{yr}^{-1}$ ) and the $\mathrm{SN}$ rate predicted by the L99 model is $\mathrm{v}_{S N} 0.06-1.3 \times 10^{-2} \mathrm{yr}^{-1}$ (average $\left.0.6 \times 10^{-2} \mathrm{yr}^{-1}\right)$. The derived star forming properties are given in Table 2. The dereddened NIR colors (Figure 3) suggest older ages (8-10 Myr) than $\operatorname{Br} \gamma$ EW. This may be due to the contamination by older starburst generations in the NIR continuum after the galaxy model was subtracted, which results in artificially low EWs of $\mathrm{Br} \gamma$. Alternatively, extinction may have been slightly underestimated.

Our analysis of the star forming properties depends somewhat on the evolutionary model which is chosen. L99 employs the newest Geneva evolutionary tracks (see e.g. Charbonnel et al. 1999 and references therein) and the Lejeune atmosphere models (Lejeune, Buser \& Cuisinier 1997) and as such may be considered the most up to date. The general result of a decaying starburst remains unchanged if one adopts the evolutionary model of Lançon \& Rocca-Volmerange (1996). A comparison between the EWs of $\mathrm{H} \beta$ in L99 and the model of Cerviño \& MasHesse (1994) further supports the model-independence of our conclusions. However, the exact age of the starburst is very model dependent - one can assume different durations or IMFs for the starburst and derive different ages.

The number of ionizing photons derived from the thermal radio emission by $\mathrm{S} 99$ is $\mathrm{N}\left(\mathrm{H}^{0}\right)=5.5 \times 10^{54} \mathrm{~s}^{-1}$, more than a magnitude larger than the $\mathrm{N}\left(\mathrm{H}^{0}\right)=2.0 \times 10^{53} \mathrm{~s}^{-1}$ that we derive from $\operatorname{Br} \gamma$. The simplest explanation for this discrepancy is an underestimation of extinction, but this would indicate $A_{V} \sim 45 \mathrm{mag}$, which is unjustified by the NIR colors. A difference between the estimates derived from $\mathrm{H} \alpha$ and the radio continuum was also noticed by $\mathrm{S} 99$, but since extinction is much higher in the optical wavelengths, they were able to explain the difference with $A_{V}=6.5$. Alternatively, it is possible that S99 have overestimated the fraction of thermal flux in the radio continuum. They suggest that more than $50 \%$ of the flux at 6 $\mathrm{cm}$ is thermal, at least in the circumnuclear region. This is a much larger fraction than what was found in the nuclear regions of normal galaxies (between $3 \%$ and 20\%; Turner \& Ho 1994).

An ISF or a short duration model seems more probable than CSFR for NGC 7771, based on the clumpy NIR continuum morphology and the observed radio spectral index, $\alpha \sim-0.5$ (S99). The L99 model does not contain radio continuum information, but the Mas-Hesse \& Kunth (1991) CSFR models cannot produce spectral indices steeper than $\sim-0.3$, while their ISF model can. The ages of the star forming regions derived by us are similar to those derived by S99 for the $K$-band regions ( $\sim 10 \mathrm{Myr})$ and the radio regions (4-8 Myr), and agree with their suggestion of several star forming generations in NGC 7771.

\section{CONCLUSIONS}

We have presented the first near-infrared emission line images of the star forming ring of NGC 7771, and interpret its star forming properties with the L99 evolutionary models. The morphology of NGC 7771 varies strongly with wavelength, which may reflect varying ages of star forming regions or several stellar generations present in the ring. Also, ellipticities and position angles of the rings differ at various wavelengths. No $\mathrm{Br} \gamma$ was detected in the nucleus, but the nucleus is a strong $\mathrm{S}(1)$ emitter. The mass of the excited $\mathrm{H}_{2}\left(M \simeq 1900 \mathrm{M}_{\odot}\right)$ is higher than in the Seyfert galaxies NGC 3079 and NGC 3227.

The EW of $\mathrm{Br} \gamma$ and the NIR morphology are consistent with an instantaneous burst of star formation 6-7 Myr ago. The observed $\mathrm{Br} \gamma \mathrm{EW}$ cannot be produced in the constant 
star formation model even with a lowered upper mass cutoff $\left(M_{u}=30 \mathrm{M}_{\odot}\right)$ without unlikely large ages, greater than 100 Myr for many regions, which are also inconsistent with other indicators (e.g. CO absorption indices, SN rates).

The United Kingdom Infrared Telescope is operated by the Joint Astronomy Centre on behalf of the U.K. Par- ticle Physics and Astronomy Research Council. Thanks are due to Tom Geballe and Thor Wold for all the help during the observations. This research has made use of the NASA/IPAC Extragalactic Database (NED), which is operated by the Jet Propulsion Laboratory, California Institute of Technology, under contract with the National Aeronautics and Space Administration.

\section{REFERENCES}

Bland-Hawthorn, J. 1995, in ASP Conf. Ser. vol 71, Tridimensional optical spectroscopic methods in astrophysics, ed. Comte, G. \& Marcelin, M. (San Francisco: ASP), 72

Blitz, L. 1991, in ASI Series 342, The Physics of Star Formation and Early Stellar Evolution, ed. Lada, C., \& Kylafis, N., (Dordrecht: Kluwer Academic Publishers), 3

Cerviño, M., \& Mas-Hesse, J. M. 1994, A\&A, 284,749

Charbonnel, C., Däppen, W., Schaerer, D., Bernasconi, P. A., Maeder, A., Meynet, G., Mowlavi, N. 1999, A\&AS, 135, 405

Condon, J. J., \& Yin, Q. F. 1990, ApJ, 357, 97

Davies, R. I., Alonso-Herrero, A., \& Ward, M. J. 1997, MNRAS, 291, 557

Fernandez, B. R., Holloway, A. J., Meaburn, J., Pedlar, A., \& Mundell, C. G. 1999, MNRAS, 305, 319

Glass, I. S., \& Moorwood, A. F. M. 1985, MNRAS, 241, 429

Golla, G., Allen, M., \& Kronberg, P. P. 1996, ApJ, 473, 244

Keel, W. C. 1996, ApJS, 106, 27

Keel, W. C. 1993, AJ, 106, 1771

Kotilainen, J. K., Reunanen, J., Laine, S., \& Ryder, S. D. 1999, A\&A, submitted

Lançon, A., \& Rocca-Volmerange, B. 1996, New Astr., 1, 215

Landini, M., Natta, A., Oliva, E., Salinari, P., \& Moorwood, A. F. M. 1984, A\&A, 134, 284

Leitherer, C., \& Heckman, T. M. 1995, ApJS, 96,9
Leitherer, C., Schaerer, D., Goldader, J. et al. 1999, ApJS, 123,3

Lejeune, T., Buser, R., \& Cuisinier, F. 1997, A\&AS, 125, 229

Mas-Hesse, J. M., \& Kunth, D. 1991, A\&AS, 88, 399

Meaburn, J., Fernandez, B. R., Holloway, A. J., Pedlar, A., Mundell, C. G., \& Geballe, T. R. 1998, MNRAS, 295, L45

Neff, S. G., \& Hutchings, J. B. 1992, A.J, 103, 1746

Nordgren, T., Chengalur, J., Salpeter, E., \& Terzian, Y. 1997, AJ, 114,77

Puxley, P. J., Doyon, R., \& Ward, M. J. 1997, ApJ, 476, 120

Puxley, P. J., Hawarden, T. G., \& Mountain, C. M. 1990, ApJ, 364, 77

Ridgway, S. E., Wynn-Williams C. G., \& Becklin, E. E. 1994, ApJ, 428,609

Sams, B. H., Genzel, R., Eckart, A., Tacconi-Garman, L., \& Hofmann, R. 1994, ApJ, 430, L33

Sanders, D. B, Scoville, N. Z., \& Soifer, B. T. 1991, ApJ, 370, 158

Smith, D. A., Haynes, M. P., Beichman, C. A., \& Gautier, T. N. 1996, ApJS, 104, 217

Smith, D. A., Herter, T., Haynes, M. P., \& Neff, S. G. 1999, ApJ, 510, 669 (S99)

Turner, J. L., \& Ho, P. T. P. 1994, ApJ, 421, 122

Ulvestad, J. S., \& Antonucci, R. R. J. 1997, ApJ, 488, 621

Veilleux, S., Kim, D.-C., Sanders, D. B., Mazzarella, J. M., \& Soifer, B. T. 1995, ApJS, 98, 171 
FIG. 1. - The $K$-band image of NGC 7771 with $\mathrm{Br} \gamma$ (black contours) and $\mathrm{H}_{2} 1-0 \mathrm{~S}(1)$ (white contours) line emission overlaid. The lowest contour $(3 \sigma)$ of $\mathrm{Br} \gamma$ is $32 \%$ of the peak $\left(5.1 \times 10^{-16} \mathrm{erg} \mathrm{s}^{-1} \mathrm{~cm}^{-2} \operatorname{arcsec}^{-2}\right)$ and other contours are at 50,70 and $85 \%$. In $\mathrm{S}(1)$, the contours are at $18,30,50,70$ and $85 \%$ of the peak luminosity $\left(7.7 \times 10^{-16} \mathrm{erg} \mathrm{s}^{-1} \mathrm{~cm}^{-2} \operatorname{arcsec}^{-2}\right)$. The arrows point to the Br $\gamma$ regions and to the nucleus mentioned in the text.

FIG. 2.- The $J-H$ color map of NGC 7771 . The highest contour is 1.0, and the contour interval is 0.1

FIG. 3.- The observed (filled circles) and dereddened (open circles) $J-H$ - and $H-K$-colors of NGC 7771 . The nucleus has been marked with a triangle. The average colors of unobscured spiral galaxies $(\langle J-H\rangle=0.75,\langle H-K\rangle=0.22$; Glass \& Moorwood 1985) are indicated by an ellipse. The average errors of the observed colors, the effects of extinction (arrow) and hot dust emission (dotted line) on the colors, and the colors predicted by the L99 model (ISF, solid line) are also indicated. The tickmarks show the fractional contribution of dust to the $K$-band emission at $10 \%$ intervals.

TABLE 1

BR $\gamma$ EMISSION REGIONS

\begin{tabular}{|c|c|c|c|c|c|c|c|c|c|c|c|c|}
\hline \multirow[b]{2}{*}{$\mathrm{n}$} & \multirow[b]{2}{*}{$\underset{\prime \prime}{\mathrm{ap}}$} & \multirow[b]{2}{*}{$\operatorname{corr}^{\mathrm{c}}$} & \multicolumn{4}{|c|}{ observed emission $^{\mathrm{a}}$} & \multicolumn{5}{|c|}{ dereddened emission $^{\mathrm{b}}$} & \multirow[b]{2}{*}{$\begin{array}{l}\mathrm{A}_{V}^{\mathrm{d}} \\
\mathrm{mag}\end{array}$} \\
\hline & & & $\begin{array}{l}\mathrm{Br} \gamma \\
10^{-15}\end{array}$ & $\begin{array}{c}\mathrm{S}(1) \\
\operatorname{ergs~\mathrm {s}^{-1}} \mathrm{cm}^{-2}\end{array}$ & $\begin{array}{l}\mathrm{J}-\mathrm{H} \\
\mathrm{mag}\end{array}$ & $\begin{array}{l}\mathrm{H}-\mathrm{K} \\
\mathrm{mag}\end{array}$ & $\begin{array}{l}\mathrm{Br} \gamma \\
10^{-15}\end{array}$ & $\begin{array}{c}\mathrm{S}(1) \\
\operatorname{ergs~s}^{-1} \mathrm{~cm}^{-2}\end{array}$ & $\frac{S(1)}{B r \gamma}$ & $\begin{array}{r}\mathrm{J}-\mathrm{H} \\
\mathrm{mag}\end{array}$ & $\begin{array}{l}\mathrm{H}-\mathrm{K} \\
\mathrm{mag}\end{array}$ & \\
\hline 1 & 2.2 & 1.52 & 0.59 & 1.23 & 0.84 & 0.33 & 0.73 & 1.54 & 2.10 & 0.59 & 0.18 & 1.1 \\
\hline 2 & 1.4 & 1.11 & 0.70 & 1.44 & 0.82 & 0.36 & 0.87 & 1.80 & 2.06 & 0.57 & 0.21 & 1.0 \\
\hline 3 & 2.2 & 1.03 & 0.91 & 1.76 & 0.91 & 0.42 & 1.13 & 2.20 & 1.94 & 0.66 & 0.27 & 2.1 \\
\hline 4 & 1.7 & 1.02 & 0.35 & 0.21 & 0.78 & 0.18 & 0.44 & 0.26 & 0.61 & 0.53 & 0.03 & 0.5 \\
\hline 5 & 1.4 & 1.01 & 0.41 & 0.54 & 0.92 & 0.42 & 0.51 & 0.68 & 1.32 & 0.67 & 0.26 & 2.2 \\
\hline 6 & 1.0 & 1.01 & 0.27 & 0.24 & 1.01 & 0.52 & 0.33 & 0.29 & 0.89 & 0.76 & 0.37 & 3.3 \\
\hline 7 & 1.4 & 1.02 & 0.62 & 0.58 & 1.06 & 0.58 & 0.77 & 0.73 & 0.95 & 0.81 & 0.42 & 3.9 \\
\hline 8 & 1.4 & 1.02 & 0.45 & 0.34 & 1.00 & 0.53 & 0.56 & 0.43 & 0.76 & 0.75 & 0.37 & 3.1 \\
\hline 9 & 1.4 & 1.06 & 0.46 & 0.32 & 0.93 & 0.47 & 0.57 & 0.40 & 0.71 & 0.67 & 0.32 & 2.2 \\
\hline $\mathrm{N}$ & 1.1 & 1.01 & - & 2.61 & 0.98 & 0.46 & - & 3.24 & - & 0.73 & 0.31 & 2.9 \\
\hline
\end{tabular}

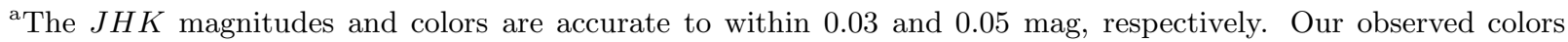
agree well with those reported by S99. For the narrowband photometry, accuracy varies from $8 \%$ (region 3 ) to $12 \%$ (region 1), with average value is $9 \%$.

${ }^{\mathrm{b}}$ Dereddened using $\mathrm{A}_{V}=2.8$

${ }^{\mathrm{c}}$ Correction factor for the velocity field and the change of wavelength across the array

${ }^{\mathrm{d}}$ Obtained from the NIR color: $\langle J-H\rangle=0.75$ (Glass \& Moorwood 1985) 
TABLE 2

StAR FORMING PROPERTIES

\begin{tabular}{|c|c|c|c|c|c|c|c|c|c|c|}
\hline \multirow[b]{2}{*}{$\mathrm{n}$} & \multirow[b]{2}{*}{$\begin{array}{c}\mathrm{N}\left(\mathrm{H}^{0}\right) \\
10^{52} \mathrm{~s}^{-1}\end{array}$} & \multirow[b]{2}{*}{$\begin{array}{c}\mathrm{EW} \\
\AA\end{array}$} & \multicolumn{4}{|c|}{ instant star formation } & \multicolumn{4}{|c|}{ constant star forming rate } \\
\hline & & & $\begin{array}{l}\text { age } \\
10^{6} \mathrm{yr}\end{array}$ & $\begin{array}{c}\text { mass } \\
10^{6} \mathrm{M}_{\odot}\end{array}$ & $\begin{array}{c}\mathrm{SFR}^{\mathrm{a}} \\
\mathrm{M}_{\odot} \mathrm{y}^{-1}\end{array}$ & $\begin{array}{c}\mathrm{v}_{S N} \\
10^{-3} \mathrm{yr}^{-1}\end{array}$ & $\begin{array}{c}10^{6} \mathrm{yr} \\
\mathrm{Myr}\end{array}$ & $\begin{array}{c}\mathrm{SFR} \\
\mathrm{M}_{\odot} \mathrm{y}^{-1}\end{array}$ & $\begin{array}{c}\operatorname{mass}^{b} \\
10^{6} \mathrm{M}_{\odot}\end{array}$ & $\begin{array}{c}\mathrm{v}_{S N} \\
10^{-3} \mathrm{yr}^{-1}\end{array}$ \\
\hline 1 & 2.14 & 18.7 & 6.37 & 12.6 & 1.98 & 11.9 & 11.8 & 0.46 & 5 & 2.3 \\
\hline 2 & 2.55 & 8.55 & 6.63 & 20.0 & 3.01 & 18.4 & 48.2 & 0.55 & 26 & 9.2 \\
\hline 3 & 3.32 & 5.46 & 6.81 & 32.2 & 4.73 & 32.6 & 176 & 0.71 & 125 & 12.5 \\
\hline 4 & 1.28 & 54.0 & 5.84 & 3.4 & 0.59 & 2.9 & 8.4 & 0.28 & 2 & 0.6 \\
\hline 5 & 1.50 & 6.58 & 6.72 & 13.6 & 2.02 & 12.5 & 100 & 0.32 & 32 & 6.7 \\
\hline 6 & 0.97 & 7.93 & 6.65 & 7.8 & 1.18 & 7.2 & 59.5 & 0.21 & 12 & 3.9 \\
\hline 7 & 2.26 & 8.91 & 6.62 & 17.4 & 2.63 & 16.1 & 43,6 & 0.49 & 21 & 9.2 \\
\hline 8 & 1.65 & 6.50 & 6.72 & 14.9 & 2.22 & 13.8 & 104 & 0.35 & 37 & 6.2 \\
\hline 9 & 1.66 & 5.76 & 6.78 & 15.8 & 2.34 & 15.5 & 149 & 0.36 & 53 & 6.9 \\
\hline
\end{tabular}

${ }^{a}$ Mass divided by age

${ }^{\mathrm{b}} \mathrm{SFR}$ multiplied by age 


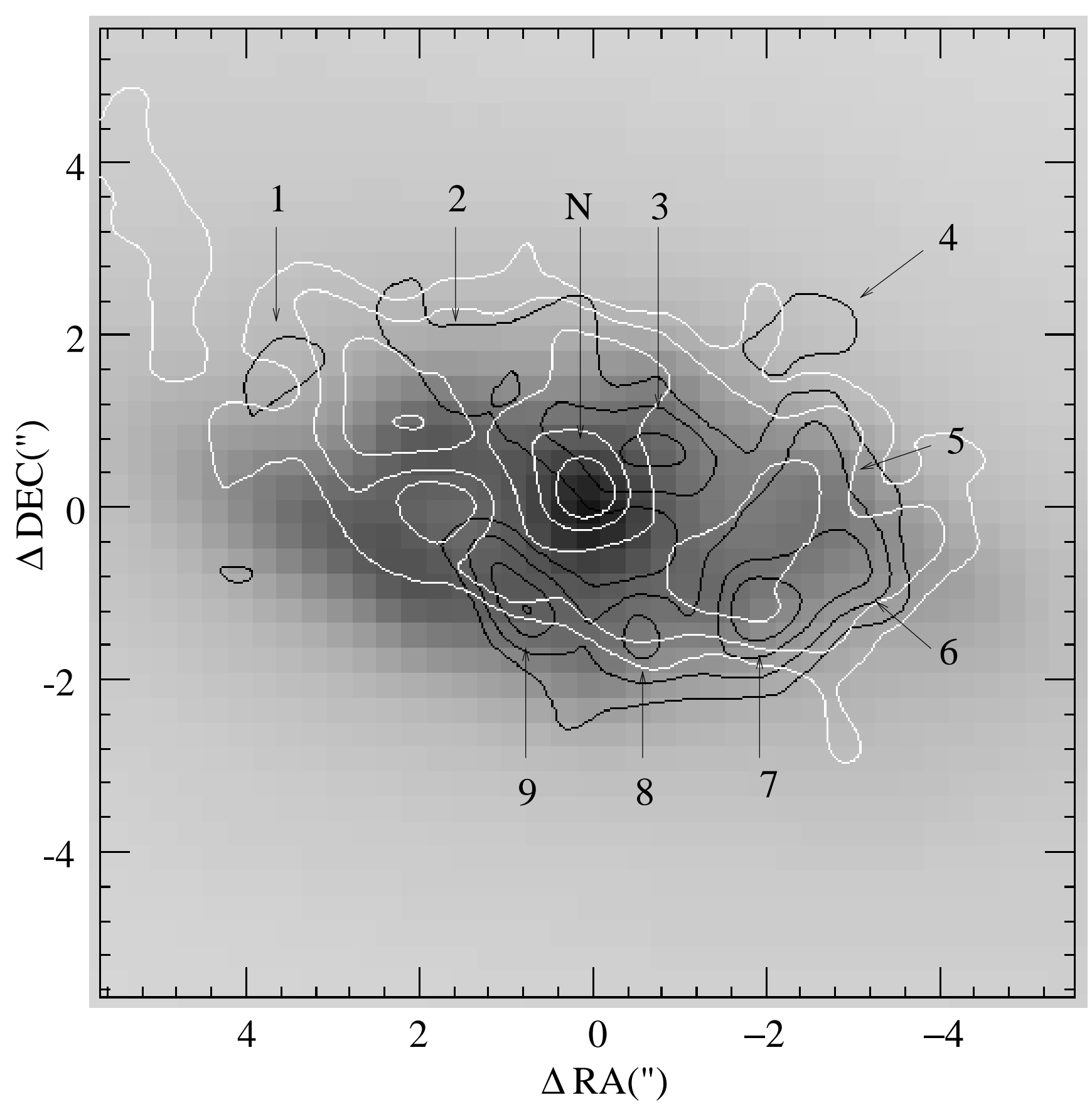




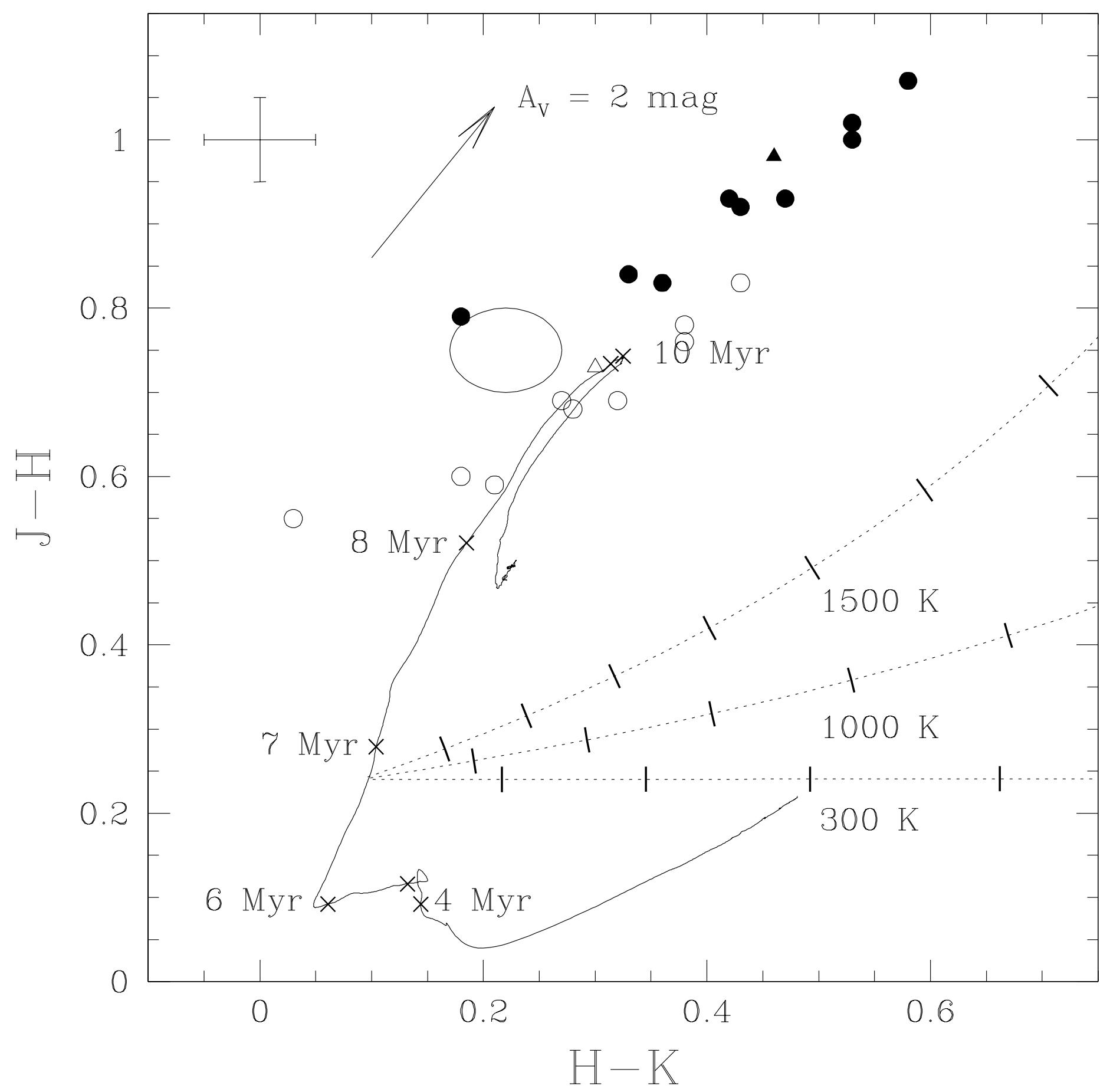

